Research Article

\title{
Disturbance Rejection Trajectory Tracking Control for an Unmanned Quadrotor Based on Hybrid Controllers
}

\author{
Xiaoming Ji $\mathbb{D}^{1}$ and Zihui Cai ${ }^{2}$ \\ ${ }^{1}$ Department of Electrical Engineering, Jiangsu College of Safety Technology, Xuzhou 221011, Jiangsu, China \\ ${ }^{2}$ Beijing Satellite Manufacturing Co., Ltd, Beijing 100000, China \\ Correspondence should be addressed to Xiaoming Ji; jxm27@163.com
}

Received 9 June 2021; Revised 19 June 2021; Accepted 1 July 2021; Published 10 July 2021

Academic Editor: L. Fortuna

Copyright (C) 2021 Xiaoming Ji and Zihui Cai. This is an open access article distributed under the Creative Commons Attribution License, which permits unrestricted use, distribution, and reproduction in any medium, provided the original work is properly cited.

\begin{abstract}
The purpose of this article is to explore a dual-loop problem regarding the trajectory tracking control of a quadrotor unmanned aerial vehicle, applying a linear active disturbance rejection and conditional integrator sliding mode controller, namely, LARCCISMC. The quadrotor system model is derived through Newton-Euler method and consists of two subsystems. The hybrid controller for position and attitude loops is constructed. An evaluation of the proposed controller is presented in comparison with the linear active disturbance rejection controller. Simulation comparisons and experimental tests illustrate that the proposed controller has a satisfied robustness and accuracy under lumped disturbances.
\end{abstract}

\section{Introduction}

Recently, with the development of automation and unmanned aerial vehicle, the researchers have focused on flight controller design to enhance the performance of the aircraft in operating autonomous flight missions. They have been applied in both civilian and military applications such as aerial photography, target location, disaster management, and agricultural care [1-3]. Quadrotors are special kinds of UAVs that are capable of vertical take-offs and landings, hovering at a low altitude, and flying in all directions. In particular, the high levels of automation, flexibility, and complexity of intelligent aerial equipment present new challenges to controller design. Significantly, the control, operation, and interaction of the quadrotors are quite challenging due to their inherently unstable, highly nonlinear, and underactuated dynamics [4]. The external disturbances in the harsh environment are inevitable to impact the stability of the quadrotors, which leads to the difficult controller design. Meanwhile, the controller is sensitive to the coupled dynamics of the quadrotors. Besides the modelled characteristics, unmodeled characteristics are another important consideration in controller design. Moreover, the existing uncertain conditions (external disturbances such as wind gusts), modelling errors (internal uncertainty), and structural parameter variation make the design of the flight control architecture particularly challenging. Consequently, it is imperative to develop robust, efficient, and high-performance control approaches capable of addressing all of the issues above and improving control performance and stability.

Recently, numerous achievements have been reported about the control methods for the quadrotors. In the closedloop control system of a microquadrotor, a linear quadratic controller is used to realize the goal of trajectory tracking control [5]. Ma et al. proposed a model predictive control method to solve the trajectory tracking problem under external disturbances [6]. A linear quadratic Gaussian with integral action is applied to stabilize a quadrotor's attitude with good results in the hover phase [7]. Ansari and Bajodah proposed a two-loop robust generalized dynamical inversion controller for quadrotor attitude and position control, which provides a robust trajectory tracking strategy subjected to model uncertainties and parametric variations [8]. Despite the fact that the aforementioned control strategies offer a 
good balance among control performance, operational costs, and computational complexity, they tend to be restricted by the predictive mathematical models' accuracy. Furthermore, the controllers are also vulnerable to lumped disturbances, where the performance of these strategies will be significantly reduced under the uncertain conditions.

Researchers have also focused attention on model-free control strategies that need no information of the model. For example, Tian et al. [9] proposed a multivariable supertwisting sliding mode control (SMC) method for finite-time attitude control of a quadrotor, and numerical simulation and experimental verification illustrate the efficiency of the proposed controller. A high-performance trajectory tracking controller using the backstepping technique is developed for the quadrotor using a disturbance observer [10]. While relying only on a nominal model and its limits, the disturbance observer can estimate disturbances. Najm and Ibraheem [11] used linear active disturbance rejection control (LADRC) method to stabilize the altitude and attitude of a quadrotor. LADRC has a linear extended disturbance observer which can reject the lumped disturbances. However, there are two control loops (attitude loop and position loop) in the quadrotor system. A single controller may not be suitable for the cascade structure. Sometimes, the outputs produced from the position loop may cause undesirable transients, bringing unexpected damage to the system's components. Therefore, approaches that consider a combination of multiple control strategies are favoured by scholars.

Ding et al. [12] combined LADRC and integral backstepping control to realize trajectory tracking of a multicopter. In the above control system, the LADRC has more advantages in rejecting the inner-loop disturbances, and the integral backstepping control can eliminate the static errors in the position loop well. Similarly, Mohd Basri et al. [13] proposed a hybrid controller combining the backstepping technique and adaptive fuzzy method to realize the complex trajectory tracking of a quadrotor, which effectively suppresses the time-varying perturbations. To stabilize the dualloop state variables of a quadrotor, a novel controller combining robust generalized dynamic inversion and adaptive nonsingular terminal sliding mode is proposed [14]. Motivated by the above controllers, we will develop a hybrid dual-loop controller for a quadrotor. The main goal in this article is to demonstrate and show an improvement in the tracking performance of a quadrotor control by a hybrid approach. The main contributions of this article are listed as follows:

(i) The principle and composition of the hybrid controller are studied. More specially, a LADRC is introduced to stabilize the position loop, and a CISMC is used to stabilize the attitude loop.

(ii) The proposed controller is investigated in terms of simulation and real-world application in the context of quadrotor trajectory tracking. To the best of the authors' knowledge, no reports on the LADRCCISMC trajectory tracking control technique for quadrotors are available until now. (iii) Good robustness is provided against various lumped disturbances, including modelling errors, external disturbances, and noise measurements.

An overview of this article can be found as follows. The quadrotor system model is presented in Section 2. In Sections 3 and 4, a hybrid controller is developed, as well as stability analysis. In Section 5, the results of simulations and experiments are presented. The main conclusions are presented in Section 6.

\section{System Description}

A quadrotor aircraft is driven by the propellers mounted at the ends of an $X$-shaped frame as shown in Figure 1. Each propeller offers thrust $F_{j}(j=1 \sim 4)$ and moment $M_{j}(j=1 \sim 4)$. The motion of the aircraft is described with two coordinate frames, i.e., the earth-fixed coordinate frame $O_{E} X_{E} Y_{E} Z_{E}(\{E\})$ and body-fixed coordinate frame $O_{B} X_{B} Y_{B} Z_{B}(\{B\})$. The symbols $\phi, \theta$, and $\psi$ indicate the Euler angles (roll, pitch, and yaw), respectively. $\omega_{j}(j=1 \sim 4)$ denotes the propeller speed.

Based on the mechanical structure and driven mechanism, the control signals of the quadrotor are calculated as follows:

$$
\left\{\begin{array}{l}
\tau_{1}=F_{1}+F_{2}+F_{3}+F_{4}, \\
\tau_{2}=\left(\frac{\sqrt{2}}{2} F_{1}-\frac{\sqrt{2}}{2} F_{2}-\frac{\sqrt{2}}{2} F_{3}+\frac{\sqrt{2}}{2} F_{4}\right) L, \\
\tau_{3}=\left(\frac{\sqrt{2}}{2} F_{1}+\frac{\sqrt{2}}{2} F_{2}-\frac{\sqrt{2}}{2} F_{3}-\frac{\sqrt{2}}{2} F_{4}\right) L, \\
\tau_{4}=M_{2}+M_{4}-M_{1}-M_{3},
\end{array}\right.
$$

where $L$ is the distance between a propeller and the mass of the aircraft and $\tau=\left[\tau_{1}, \tau_{2}, \tau_{3}, \tau_{4}\right]^{T}$ is the control signal.

The rigid body dynamical model of the quadrotor is derived by applying Newton-Euler's equations [15]:

$$
\left\{\begin{array}{l}
m \ddot{\mathbf{p}}=\mathbf{R}_{E}^{B}\left(\tau_{1} \mathbf{Z}_{B}\right)-m g \mathbf{Z}_{I}+\mathbf{F}_{D}, \\
\mathbf{I} \ddot{\mathbf{q}}=\left[\begin{array}{lll}
\tau_{2} & \tau_{3} & \tau_{4}
\end{array}\right]^{T}-\dot{\mathbf{q}}^{T} \mathbf{I} \dot{\mathbf{q}},
\end{array}\right.
$$

where $m$ is the mass of the aircraft, $g$ is the gravitational acceleration, $\mathbf{q}=[\phi, \theta, \psi]^{T}$ is the Euler angle vector, $\mathbf{p}=$ $[x, y, z]^{T}$ is the position vector, $\mathbf{I}=\operatorname{diag}\left(I_{x}, I_{y}, I_{z}\right)$ is the rotational inertia, and $\mathbf{Z}_{B}$ and $\mathbf{Z}_{I}$ are the unit vector in the body-fixed coordinate frame and earth-fixed coordinate frame, respectively. The matrix $\mathbf{R}_{E}^{B}$ maps from earth-fixed coordinates to body-fixed coordinates, which is governed by yaw-pitch-yaw Euler angles:

$$
\mathbf{R}_{E}^{B}=\left[\begin{array}{ccc}
c \theta c \psi & s \phi s \theta c \psi-c \phi s \psi & c \phi s \theta c \psi+s \phi s \psi \\
c \theta s \psi & s \phi s \theta s \psi+c \phi c \psi & c \phi s \theta s \psi-s \phi c \psi \\
-s \theta & s \phi c \theta & c \phi c \theta
\end{array}\right],
$$

where $s \alpha$ and $c \alpha$ are the simplified forms of $\cos \alpha$ and $\sin \alpha$. 


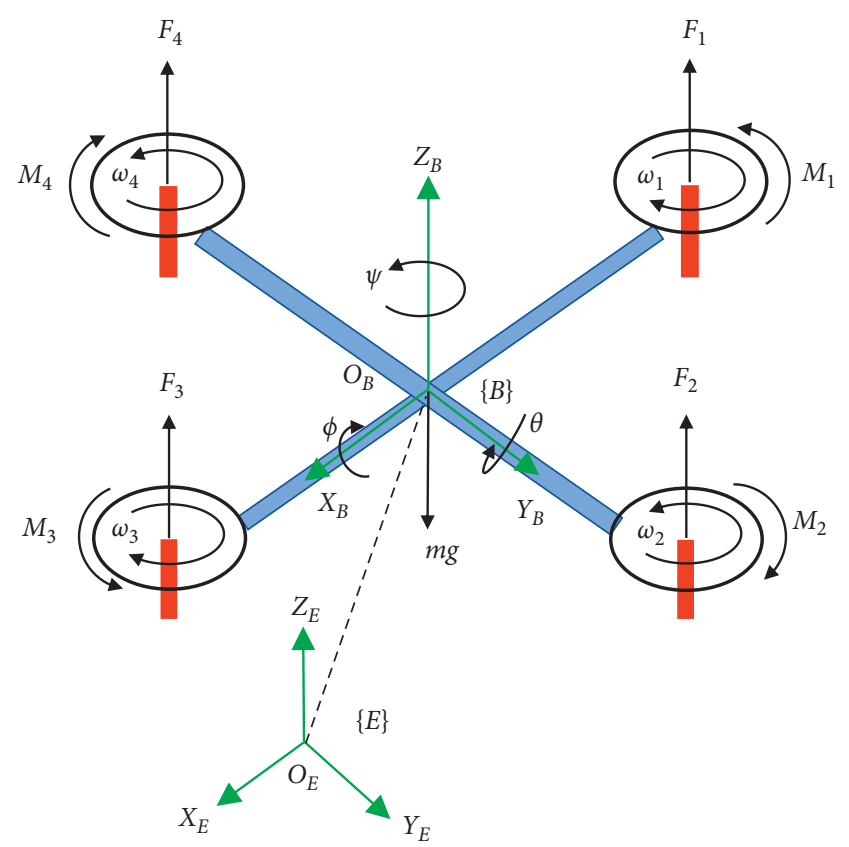

FIgURE 1: Free body diagram of quadrotor.

Furthermore, model (2) can be described into translational and rotational submodels that denote its linear and angular positions, respectively:

$$
\left\{\begin{array}{l}
\ddot{x}=\frac{\tau_{1}(c \psi s \theta c \phi+s \psi s \phi)}{m-k_{x} \dot{x}|\dot{x}| / m}, \\
\ddot{y}=\frac{\tau_{1}(s \psi s \theta c \phi-c \psi s \phi)}{m-k_{y} \dot{y}|\dot{y}| / m}, \\
\ddot{z}=\frac{\tau_{1} c \phi c \theta}{m-g-k_{z} \dot{z}|\dot{z}| / m}, \\
\ddot{\phi}=\frac{\left[\tau_{2}+\dot{\theta} \dot{\psi}\left(I_{y}-I_{z}\right)\right]}{I_{x}}, \\
\ddot{\theta}=\frac{\left[\tau_{3}+\dot{\phi} \dot{\psi}\left(I_{z}-I_{x}\right)\right]}{I_{y}}, \\
\ddot{\psi}=\frac{\left[\tau_{4}+\dot{\phi} \dot{\theta}\left(I_{x}-I_{y}\right)\right]}{I_{z}},
\end{array}\right.
$$

where $k_{x}, k_{y}$, and $k_{z}$ are the air drag coefficient. The mathematical relation between the control inputs and angular velocities is given by

$$
\left[\begin{array}{c}
\tau_{1} \\
\tau_{2} \\
\tau_{3} \\
\tau_{4}
\end{array}\right]=\left[\begin{array}{cccc}
k_{t} & k_{t} & k_{t} & k_{t} \\
\frac{\sqrt{2}}{2} k_{t} L & -\frac{\sqrt{2}}{2} k_{t} L & -\frac{\sqrt{2}}{2} k_{t} L & \frac{\sqrt{2}}{2} k_{t} L \\
\frac{\sqrt{2}}{2} k_{t} L & \frac{\sqrt{2}}{2} k_{t} L & -\frac{\sqrt{2}}{2} k_{t} L & -\frac{\sqrt{2}}{2} k_{t} L \\
-k_{m} & k_{m} & -k_{m} & k_{m}
\end{array}\right]\left[\begin{array}{c}
\omega_{1}^{2} \\
\omega_{2}^{2} \\
\omega_{3}^{2} \\
\omega_{4}^{2}
\end{array}\right],
$$

where $k_{t}$ and $k_{m}$ are termed as thrust coefficient and moment coefficient, respectively, and $L$ is the distance between the center of the propeller hub and the quadrotor mass counter.

The thrusts and moments applied to the quadrotor can be calculated by the propeller speed as follows:

$$
\left\{\begin{array}{l}
F_{j}=\rho_{a} k_{t} A R^{2} \omega_{j}^{2}, \\
M_{j}=\rho_{a} k_{m} A R^{3} \omega_{j}^{2},
\end{array}\right.
$$

where $\rho_{a}$ denotes the density of air, $A$ represents the disk area of the propeller, and $R$ is the radius of the propeller.

The dynamic model (4) can be simplified when the quadrotor flies in hover or in low-speed cruise [15]. At this case, $\sin \theta \approx \theta, \sin \phi \approx \phi, \cos \theta \approx 1$, and $\cos \phi \approx 1$. Meanwhile, due to the fact that the small rotary inertia and symmetrical structure of the quadrotor result in a small and insignificant term $\dot{q}^{T} \mathbf{I} \dot{q}[16]$, hence, model (4) can be reduced into

$$
\left\{\begin{array}{l}
m \ddot{x}=\tau_{1}(\theta \cos \psi+\phi \sin \psi), \\
m \ddot{y}=\tau_{1}(\theta \sin \psi-\phi \cos \psi), \\
m \ddot{z}=\tau_{1}-m g, \\
\ddot{\phi}=\frac{\tau_{2}}{\tau_{2}} \\
\ddot{\theta}=\frac{\tau_{3}}{I_{y}} \\
\ddot{\psi}=\frac{\tau_{4}}{I_{z}} .
\end{array}\right.
$$

The control structure of the quadrotor is illustrated in Figure 2. The system is composed of two loops, a position loop and an attitude loop. Furthermore, the closed-loop system has four channels: altitude channel, position channel, 


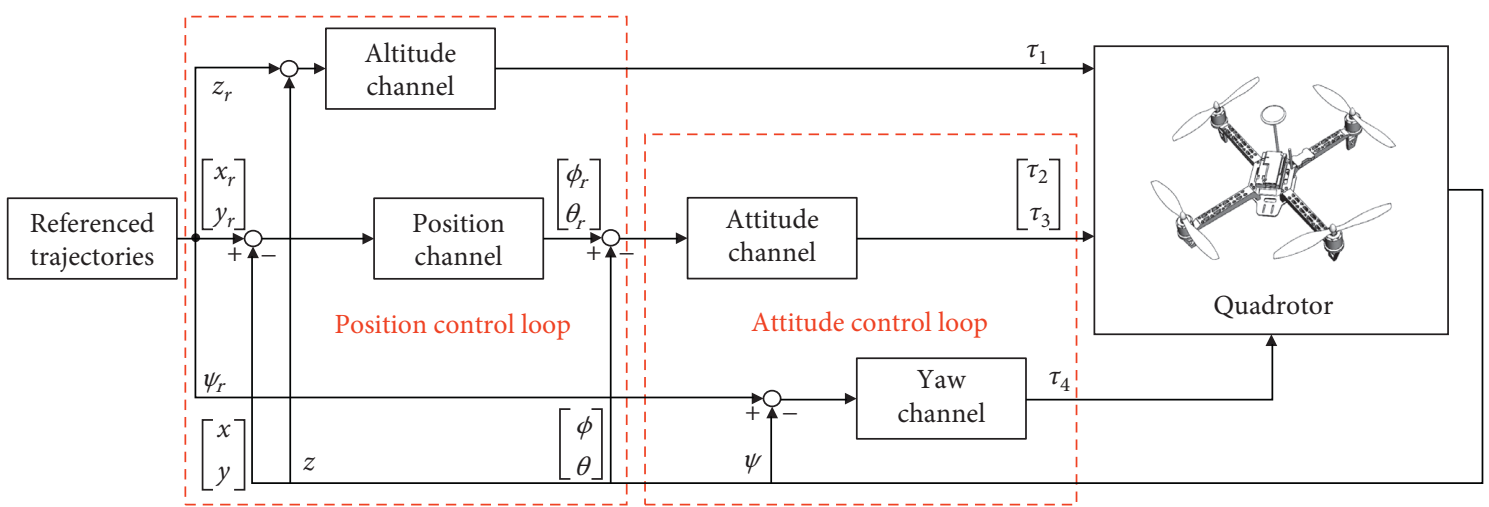

Figure 2: Control structure of the quadrotor.

attitude channel, and yaw channel. It is evident that the position channel and the attitude channel are interdependent; namely, the outputs of the position channel are the reference of the attitude channel. The controller design in our work requires no information of the dynamical model and relies only on input and output data. Besides, each state variable in (7) is related to the control input as a second-order derivative.

\section{Position Loop Control Strategy}

The purpose of the position control loop is to stabilize the states $x, y, z$ and ensure their tracking errors approach zeros. However, there are lumped disturbances in the position loop which can reduce the control performance. LADRC incorporates lumped disturbances into system states, removes disturbances by a linear extended state observer (LESO), and uses feedback control law so that no detailed and accurate mathematical modelling is required. Consider a secondorder system is in its general form as [17]

$$
\left\{\begin{array}{l}
\ddot{x}_{s}=f_{s}\left(x_{s}, \dot{x}_{s}, w_{s}, t\right)+b(t) u, \\
y_{s}=x_{s}
\end{array}\right.
$$

where $u$ and $y_{s}$ are system input and system output, respectively, $f_{s}$ are the lumped disturbances, $x_{s}$ are state variables, and $b(t)$ are the control terms.

Remark 1 (see [18]). The lumped disturbances $f_{L}$ are another type of the term $f_{s}\left(x_{s}, \dot{x}_{s}, w_{s}, t\right)$ that includes parametric variations, environmental disturbances, and complex unmodeled features [18].

Assumption 1. Suppose that $f_{L}$ is bounded and differentiable. As a result, one gets $\left\|f_{L}\right\|<\infty,\left\|\dot{f}_{L}\right\|<\infty$, and their bounds satisfies $\sup _{t>0}\left\|f_{L}\right\|=f_{b}$ and $\sup _{t>0}\|\dot{f}\|=h$.

Rewrite (8) as

$$
\left\{\begin{array}{l}
x_{s}=f+b(t) u, \\
y_{s}=x_{s} .
\end{array}\right.
$$

A differential equation can be derived from the above equation by rewriting it as follows:

$$
\left\{\begin{array}{l}
\dot{x}_{s_{1}}=x_{s_{2}}, \\
\dot{x}_{s_{2}}=x_{s_{3}}+b_{0} u, \\
\dot{x}_{s_{3}}=h \\
y_{s}=x_{s_{1}},
\end{array}\right.
$$

with $x_{s_{3}}=f_{L}$ added as an augmented state, $h=\dot{f}_{L}$. Transform model (9) into state-space form as

$$
\left\{\begin{array}{l}
\dot{x}_{s}=\mathbf{A}_{s} \mathbf{x}_{s}+\mathbf{B}_{s} \mathbf{u}_{s}+\mathbf{E}_{s} \mathbf{h}_{s}, \\
\mathbf{y}_{s}=\mathbf{C}_{s} \mathbf{x}_{d},
\end{array}\right.
$$

where

$$
\begin{aligned}
& \mathbf{A}_{s}=\left[\begin{array}{lll}
0 & 1 & 0 \\
0 & 0 & 1 \\
0 & 0 & 0
\end{array}\right], \\
& B_{s}=\left[\begin{array}{l}
0 \\
b_{0} \\
0
\end{array}\right], \\
& \mathbf{C}_{s}=\left[\begin{array}{lll}
1 & 0 & 0
\end{array}\right], \\
& \mathbf{E}_{s}=\left[\begin{array}{l}
0 \\
0 \\
1
\end{array}\right] .
\end{aligned}
$$

Remark 2. Compared with (7), system (9) represents a general class of systems since it is not limited to the integralchained form.

Then, we design the following disturbance observer, which is called LESO [19], to estimate the state that contains the lumped disturbances:

$$
\left\{\begin{array}{l}
\dot{z}_{s}=\mathbf{A}_{s} \mathbf{z}_{s}+\mathbf{B}_{s} \mathbf{u}_{s}+\mathbf{L}_{s}\left(\mathbf{y}_{s}-\widehat{y}_{s}\right), \\
\hat{y}_{s}=\mathbf{C}_{s} \mathbf{z}_{s},
\end{array}\right.
$$

where $\mathbf{z}_{s}=\left[z_{1}, z_{2}, z_{3}\right]$ denotes the estimation of the state $\left[x_{s 1}, x_{s 2}, f_{L}\right]$. The observer gain $\mathbf{L}_{s}$ can be calculated as 


$$
\mathbf{L}_{s}=\left[\begin{array}{l}
l_{1} \\
l_{2} \\
l_{3}
\end{array}\right]=\left[\begin{array}{c}
\alpha_{1} \omega_{o} \\
\alpha_{2} \omega_{o}^{2} \\
\alpha_{3} \omega_{o}^{3}
\end{array}\right],
$$

where $\omega_{o}>0$ denotes the observer bandwidth. $\alpha_{1}, \alpha_{2}$, and $\alpha_{3}$ are selected to ensure the eigenvalues of $\left(\mathbf{A}_{s}-\mathbf{L}_{s} \mathbf{C}_{s}\right)$ are in the left complex plane. The characteristic equation can be given as

$$
\lambda(s)=s^{3}+\alpha_{1} s^{2}+\alpha_{2} s+\alpha_{3}=\left(s+\omega_{o}\right)^{3} .
$$

From equation (15), the coefficients are calculated as

$$
\begin{aligned}
& \alpha_{1}=3 \\
& \alpha_{2}=3 \\
& \alpha_{3}=1 .
\end{aligned}
$$

Remark 3. All the eigenvalues $\lambda(s)$ of $\mathbf{A}_{s}$ are in the left halfplane, and the LESO is a bounded-input bounded-output (BIBO) system. It is necessary to select an appropriate observer gain $\mathbf{L}_{s}$ to ensure $\mathbf{A}_{s}$ is Hurwitz [20].

Remark 4. Suppose the derivative of $\dot{f}_{L}$ in (10) meets the condition $\lim \left\|\dot{f}_{L}\right\|=0$; the estimation error in (13) will approach $\stackrel{t \rightarrow}{\mathrm{ero}}$ asymptotically.

The disturbance observer tracks the system states with appropriate observer bandwidth and yields $z_{1}(t) \longrightarrow \hat{y}(t)$, $z_{2}(t) \longrightarrow \dot{\hat{y}}(t)$, and $z_{3}(t) \longrightarrow f_{L}$.

According to the feedback linearization method, we can offset the total disturbance by simply defining the controller as

$$
u=\frac{u_{0}-z_{3}}{b_{0}}
$$

where we need to determine the error feedback $u_{0}$ and by substituting (17) into system (10), one has

$$
\ddot{y}=f_{L}-z_{3}+u_{0},
$$

where $r_{e}$ is the reference. The estimation error can be ignored if the disturbance observer is approximately treated as an ideal observer. Then, the relationship between $y$ and $u_{0}$ becomes a simple linear double-integrator:

$$
\ddot{y} \approx u_{0} \text {. }
$$

Here, the lumped disturbances are estimated and eliminated. And the control law $u_{0}$ is

$$
u_{0}=\lambda_{1}\left(r_{e}-z_{1}\right)-\lambda_{2} z_{2}
$$

A second-order plant also has the following transfer function:

$$
G_{2}=\frac{\lambda_{1}}{s^{2}+\lambda_{2} s+\lambda_{1}}
$$

Here, the gains can be calculated as [21]

$$
\begin{aligned}
& \lambda_{1}=\omega_{c}^{2}, \\
& \lambda_{2}=2 \omega_{c} .
\end{aligned}
$$

Next, the stability of LADRC will be proved.

The tracking errors of the position loop are defined as follows:

$$
\mathbf{e}=\mathbf{x}_{s}-\mathbf{z}_{s} .
$$

The error matrix is obtained by subtracting (10) from (9):

$$
\dot{e}=\mathbf{A}_{s} \mathbf{e}+\mathbf{E}_{s} \mathbf{h}_{s}
$$

where

$$
\mathbf{A}_{e}=\mathbf{A}_{s}-\mathbf{L}_{s} \mathbf{C}_{s}=\left[\begin{array}{ccc}
-l_{1} & 1 & 0 \\
-l_{2} & 0 & 1 \\
-l_{3} & 0 & 0
\end{array}\right] .
$$

Obviously, the LESO is a bounded-input boundedoutput (BIBO) stable system since all characteristic polynomial roots are located in the left half-plane.

Theorem 1. The LADRC design process from (8)-(22) leads to a BIBO stable closed-loop system since the control law and LESO are stable.

Recalling (11) (14), one has

$$
\begin{aligned}
& u=\frac{1}{b_{0}}\left[\begin{array}{lll}
-\lambda_{1} & -\lambda_{2} & -1
\end{array}\right]\left[\begin{array}{c}
z_{1}-r_{e} \\
z_{2} \\
z_{3}
\end{array}\right] \\
& =\mathbf{Q} \mathbf{z}_{s}-\frac{1}{b_{0}}\left[\begin{array}{c}
r_{e} \\
0 \\
0
\end{array}\right]=\mathbf{Q} \mathbf{z}_{s}-\mathbf{G}
\end{aligned}
$$

where $\mathbf{Q}=\left(1 / b_{0}\right)\left[\begin{array}{lll}-\lambda_{1} & -\lambda_{2}-1\end{array}\right]$.

Rewriting the closed-loop system, one gets

$$
\begin{aligned}
\dot{x}_{s} & =\mathbf{A}_{s} \mathbf{x}_{s}+\mathbf{B}_{s} \mathbf{u}_{s}+\mathbf{E}_{s} \mathbf{h}_{s}=\mathbf{A}_{s} \mathbf{x}_{s}+\mathbf{B}_{s} \mathbf{Q} \mathbf{z}_{s}-\mathbf{B}_{s} \mathbf{G}+\mathbf{E}_{s} \mathbf{h}_{s}, \\
\dot{z}_{s} & =\mathbf{A}_{s} \mathbf{z}_{s}+\mathbf{B}_{s} \mathbf{u}_{s}+\mathbf{L}_{s}\left(\mathbf{y}_{s}-\hat{y}_{s}\right) \\
& =\mathbf{L}_{s} \mathbf{C}_{s} \mathbf{x}_{s}+\left(\mathbf{A}_{s}-\mathbf{L}_{s} \mathbf{C}_{s}+\mathbf{B}_{s} \mathbf{Q}\right) \mathbf{z}_{s}-\mathbf{B}_{s} \mathbf{G} .
\end{aligned}
$$

The above system can be written into a state-space formula as follows:

$$
\left[\begin{array}{c}
\dot{x}_{s} \\
\dot{z}_{s}
\end{array}\right]=\left[\begin{array}{cc}
\mathbf{A}_{s} & \mathbf{B}_{s} \mathbf{Q} \\
\mathbf{L}_{s} \mathbf{C}_{s} & \mathbf{A}_{s}-\mathbf{L}_{s} \mathbf{C}_{s}+\mathbf{B}_{s} \mathbf{Q}
\end{array}\right]\left[\begin{array}{c}
\mathbf{x}_{s} \\
\mathbf{z}_{s}
\end{array}\right]+\left[\begin{array}{cc}
-\mathbf{B}_{s} & \mathbf{E}_{s} \\
-\mathbf{B}_{s} & \mathbf{0}
\end{array}\right]\left[\begin{array}{c}
\mathbf{G}_{s} \\
\mathbf{h}_{s}
\end{array}\right] .
$$

The controller is BIBO stable if the eigenvalues of (28) are in the left half-plane. It is obvious that $\mathbf{h}_{s}$ is bounded since $f_{L}$ is differentiable. 
According to the design concept of the LADRC, there are four independent controllers in the position loop, which is depicted in Figure 3.

\section{Attitude Loop Controller Designs}

In the attitude loop, the control goal is to stabilize the states $\phi, \theta, \psi$ and ensure their tracking errors approach zeros. The CISMC derived from SMC is robust, accurate, and easier to implement when compared to other controllers [22]. The integral term in CISMC can increase the accuracy of static error and hold the transient response. Consider a secondorder dual-integral system:

$$
\left\{\begin{array}{l}
\ddot{x}_{a}=F_{a}+b u \\
y_{a}=x_{a}
\end{array}\right.
$$

where $x_{a}$ denotes the system variable, $u$ is the control input, $y_{a}$ is the system output, $b$ is the control gain, and $F_{a}$ is the system function.

Design sliding mode surface as

$$
s=k_{0} \sigma+k_{1} e+\dot{e},
$$

where $e$ and $\dot{e}$ are the tracking error and its derivative, $k_{0}$ and $k_{1}$ are positive numbers, and $\sigma$ denotes the output of the "conditional integrator" which is described as follows:

$$
\dot{\sigma}=-k_{0} \sigma+\mu \tanh \left(\frac{s}{\mu}\right) .
$$

where $\tanh (\cdot)$ is the hyperbolic tangent function which can degree the chattering well [23] and $\mu$ is the boundary and results from the continuous approximation of the hyperbolic tangent function. The CISMC by means of the conditional integrator ensures an improvement in transient response of ISMC and recovers the SMC performance without chattering.

Since $|s(t)| \geq \mu$, the integral loop (31) will be converted into an exponential stability system with external inputs $\pm \mu$. Since $|s(t)|<\mu$, the integral loop (31) will be converted into an error function:

$$
\dot{\sigma}=k_{1} e+\dot{e} .
$$

A conditional integral sliding mode controller can be introduced to provide robust regulation:

$$
v=-k \tanh \left[\frac{s}{\mu}\right],
$$

where $k>0$ represents a control gain which is selected sufficiently large to suppress uncanceled terms in $\dot{s}$ and $\mu$ is selected sufficiently small to recover the performance of ideal SMC without an integrator.

The stability analysis of the conditional integral sliding mode controller will be proved as follows.

Substituting (29), (31), and (32) into the derivative of the sliding mode surface, one gets

$$
\dot{s}=\Delta-k \tanh \left(\frac{s}{\mu}\right)
$$

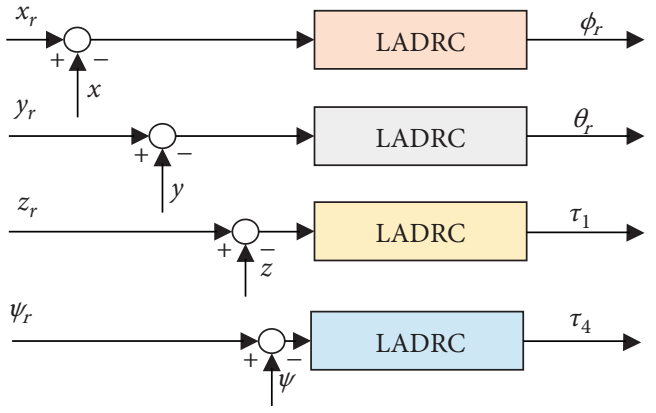

FIgURE 3: Control structure of the position loop using LADRC.

where $\Delta=-k_{0} \sigma+k_{0} \mu \tanh (s / \mu)+k_{1} \dot{e}+f+b u-\ddot{x}_{a r}$ and $\ddot{x}_{a r}$ denotes the referenced signals.

Consider a Lyapunov function:

$$
V=\frac{1}{2} s^{2},
$$

and its first derivative is

$$
\dot{V}=s \dot{s} .
$$

Substituting (34) into the above formula, one has

$$
\dot{V}=s\left[\Delta-k \tanh \left(\frac{s}{\mu}\right)\right] \leq|s||\Delta|-k s \tanh \left(\frac{s}{\mu}\right) .
$$

Suppose the error and its derivative are bounded and satisfy that $|s| \leq c(c>\mu)$; then (37) can be rewritten as

$$
\left.\dot{V} \leq|s||\Delta|-k s \tanh \left(\frac{s}{\mu}\right)\right] \leq(|\Delta|-k)|s| .
$$

Select an appropriate control gain $k$ to satisfy the condition

$$
k \geq \rho+\max (|\Delta|),
$$

where $\rho$ is a positive number. In this article, we select $\rho=1.5$.

Combining (38) and (39), we can obtain that $\dot{V} \leq 0$. This implied that the tracking error of the system will asymptotically decrease by using the proposed control law CISMC.

Remark 5 (see [24]). In equation (39), the smaller boundary $\Delta$ can be obtained by selecting the larger parameters $k$. The larger parameters $k$ are chosen, the smaller boundary $\Delta$ will be reached [24].

With the design concept of CISMC, the attitude controllers can be obtained. The tracking errors of the attitude loop are expressed as

$$
\left[\begin{array}{c}
e_{1}^{\phi} \\
e_{1}^{\theta} \\
e_{1}^{\psi}
\end{array}\right]=\left[\begin{array}{c}
\phi-\phi_{d} \\
\theta-\theta_{d} \\
\psi-\psi_{d}
\end{array}\right] .
$$

The conditional integrator sliding mode surfaces deduced by the tracking errors are given using (30): 


$$
\begin{aligned}
& {\left[\begin{array}{c}
s_{\phi} \\
s_{\theta} \\
s_{\psi}
\end{array}\right]=\left[\begin{array}{c}
k_{0}^{\phi} \sigma_{\phi}+k_{1}^{\phi} e_{1}^{\phi}+\dot{e}_{1}^{\phi} \\
k_{0}^{\theta} \sigma_{\theta}+k_{1}^{\theta} e_{1}^{\theta}+\dot{e}_{1}^{\theta} \\
k_{0}^{\psi} \sigma_{\psi}+k_{1}^{\psi} e_{1}^{\psi}+\dot{e}_{1}^{\psi}
\end{array}\right],} \\
& {\left[\begin{array}{c}
\dot{\sigma}_{\phi} \\
\dot{\sigma}_{\theta} \\
\dot{\sigma}_{\psi}
\end{array}\right]=\left[\begin{array}{c}
-k_{0}^{\phi} \sigma_{\phi}+\mu_{\phi} \tanh \left(\frac{s_{\phi}}{\mu_{\phi}}\right) \\
-k_{0}^{\theta} \sigma_{\theta}+\mu_{\theta} \tanh \left(\frac{s_{\phi}}{\mu_{\phi}}\right) \\
-k_{0}^{\psi} \sigma_{\psi}+\mu_{\psi} \tanh \left(\frac{s_{\psi}}{\mu_{\psi}}\right)
\end{array}\right] .}
\end{aligned}
$$

The first-order derivative of the sliding mode surfaces can be calculated as

$$
\left[\begin{array}{c}
\dot{s}_{\phi} \\
\dot{s}_{\theta} \\
\dot{s}_{\psi}
\end{array}\right]=\left[\begin{array}{c}
k_{0}^{\phi} \dot{\sigma}_{\phi}+k_{1}^{\phi} \dot{e}_{1}^{\phi}+\ddot{e}_{1}^{\phi} \\
k_{0}^{\theta} \dot{\sigma}_{\theta}+k_{1}^{\theta} \dot{e}_{1}^{\theta}+\ddot{e}_{1}^{\theta} \\
k_{0}^{\psi} \dot{\sigma}_{\psi}+k_{1}^{\psi} \dot{e}_{1}^{\psi}+\ddot{e}_{1}^{\psi}
\end{array}\right] .
$$

The control laws of the attitude loop can be obtained by synthesizing (7), (42), and (43):

$$
\begin{aligned}
& {\left[\begin{array}{c}
U_{2} \\
U_{3} \\
U_{4}
\end{array}\right]=\left[\begin{array}{c}
I_{x}\left[\ddot{\phi}_{r}+\left(k_{0}^{\phi}\right)^{2} \sigma_{\phi}-k_{0}^{\phi} \mu_{\phi} \tanh \left(\frac{s_{\phi}}{\mu_{\phi}}\right)-k_{1}^{\phi} \dot{e}_{1}^{\phi}+v_{\phi}\right] \\
I_{y}\left[\ddot{\theta}_{r}+\left(k_{0}^{\theta}\right)^{2} \sigma_{\theta}-k_{0}^{\theta} \mu_{\theta} \tanh \left(\frac{s_{\phi}}{\mu_{\phi}}\right)-k_{1}^{\theta} \dot{e}_{1}^{\theta}+v_{\theta}\right] \\
I_{z}\left[\ddot{\psi}_{r}+\left(k_{0}^{\psi}\right)^{2} \sigma_{\psi}-k_{0}^{\psi} \mu_{\psi} \tanh \left(\frac{s_{\psi}}{\mu_{\psi}}\right)-k_{1}^{\psi} \dot{e}_{1}^{\psi}+v_{\psi}\right]
\end{array}\right],} \\
& {\left[\begin{array}{c}
v_{\phi} \\
v_{\theta} \\
v_{\psi}
\end{array}\right]=\left[\begin{array}{l}
-k_{\phi} \tanh \left(\frac{s_{\phi}}{\mu_{\phi}}\right) \\
-k_{\theta} \tanh \left(\frac{s_{\phi}}{\mu_{\phi}}\right) \\
-k_{\psi} \tanh \left(\frac{s_{\psi}}{\mu_{\psi}}\right)
\end{array}\right] .}
\end{aligned}
$$

The control structure of the attitude loop using CISMC is depicted in Figure 4.

\section{Simulation and Experimental Results}

5.1. Simulation Results. In this section, a simulator developed with the MATLAB/SIMULINK environment has been used to test the proposed hybrid controller for a quadrotor system. The main parameters of the quadrotor

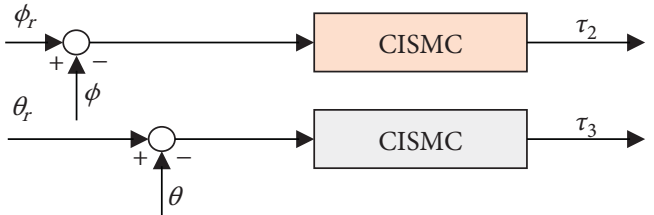

Figure 4: Control structure of the attitude loop using CISMC.

are $m=1.515 \mathrm{~kg}, I_{x}=I_{y}=0.0211 \mathrm{kgm}^{2}, I_{z}=0.0366 \mathrm{kgm}^{2}$, $L=0.63 \mathrm{~m}, \quad k_{t}=1.681 \times 10^{-5}, \quad k_{m}=2.783 \times 10^{-7}$, $\rho_{a}=1.29 \mathrm{~kg} / \mathrm{m}^{3}, A=0.17 \mathrm{~m}^{2}$, and $R=0.23 \mathrm{~m}$. It should be noted that the structural parameters can be identified by direct and indirect measurement approaches $[25,26]$, and these parameters are derived from the actual quadrotor. The purpose of the simulation is to find suitable parameters for the controller using adaptive fruit fly optimization algorithm (AFOA) [27]. The references using step signals are set as $[x, y, z]=[10 \mathrm{~m}, 10 \mathrm{~m}, 10 \mathrm{~m}]$ and $[\phi, \theta, \psi]=\left[60^{\circ},-60^{\circ}, 30^{\circ}\right]$. During parameter tuning, the attitude loop is tuned first because the position loop cannot work independently. The tuned controller parameters of the attitude loop are applied for the position loop. In addition, an improved error function [28] is introduced as an evaluation index to evaluate the optimized result:

$$
F(t)=\alpha_{1} \int_{0}^{\infty} t|e(t)| \mathrm{d} t+\alpha_{2} \int_{0}^{\infty}(u(t))^{2} \mathrm{~d} t+\alpha_{3} t_{s}+\alpha_{4} o,
$$

where $e(t)$ denotes the tracking error of the system state, $t_{s}$ denotes the settling time, $o_{s}$ denotes the overshoot, and $\alpha_{1}, \alpha_{2}, \alpha_{3}$, and $\alpha_{4}$ are the weight factors.

Lumped disturbances simulated by random signals are added to the output ports of the position loop. The simulation time of the parameters tuning in attitude loop and position loop is $5 \mathrm{~s}$, and sampling time is $1 \mathrm{~ms}$. The simulation results are summarized in Figures 5-8. We observe that the AFOA can help LADRC-CISMC to find suitable control parameters. More specifically, Figure 5 shows the system's attitude response, while the static tracking errors approach zero. It indicates the LADRC-CISMC presents a good tracking performance during the transient response. Figure 7 shows the system's position response, wherein $x, y, z$ track the reference well despite existing lumped disturbances. The results demonstrate that the LADRC-CISMC is capable of superior performance under lumped disturbances. Figures 6 and 8 show the iteration curves of AFOA. As can be observed, the IITAE index has the ability to perform in-depth data mining, whereas the AFOA is capable of helping LADRC-CISMC to obtain desired tracking performance. Furthermore, the simulation provides a set of probable control parameters that can be manually adjusted during the experimental testing phase.

The optimized control parameters of the proposed controller by AFOA are listed in Table 1.

5.2. Experimental Results. In this section, an experimental flight test has been conducted to validate the performance of 


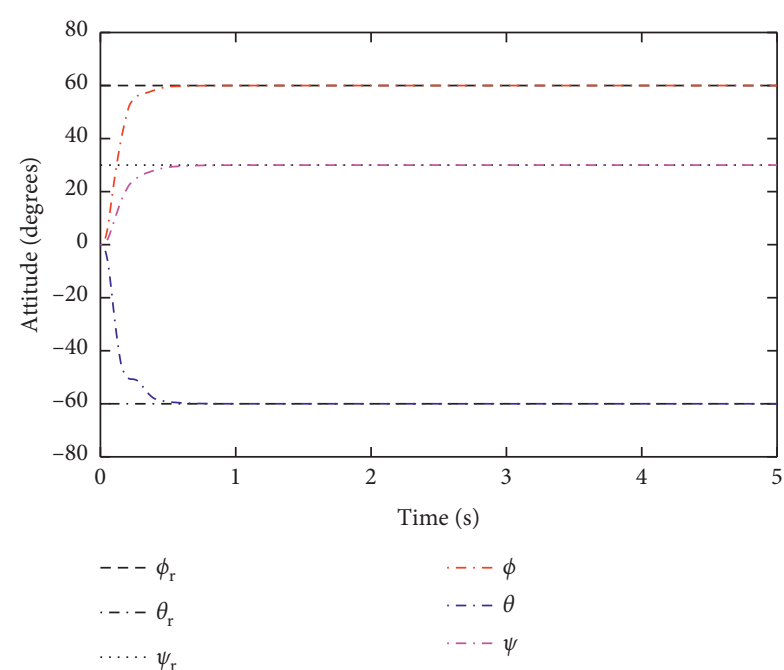

Figure 5: Step response of the attitude loop.

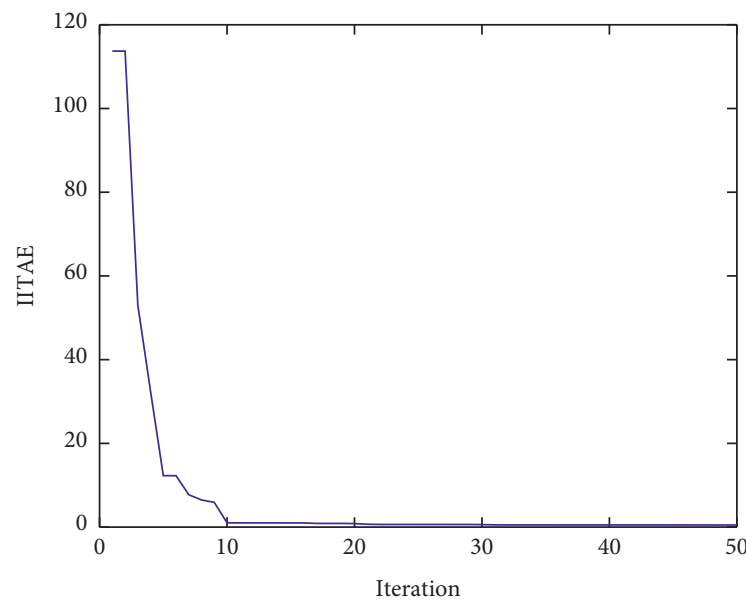

FIgURE 6: Iteration curve of the LADRC of the attitude loop.

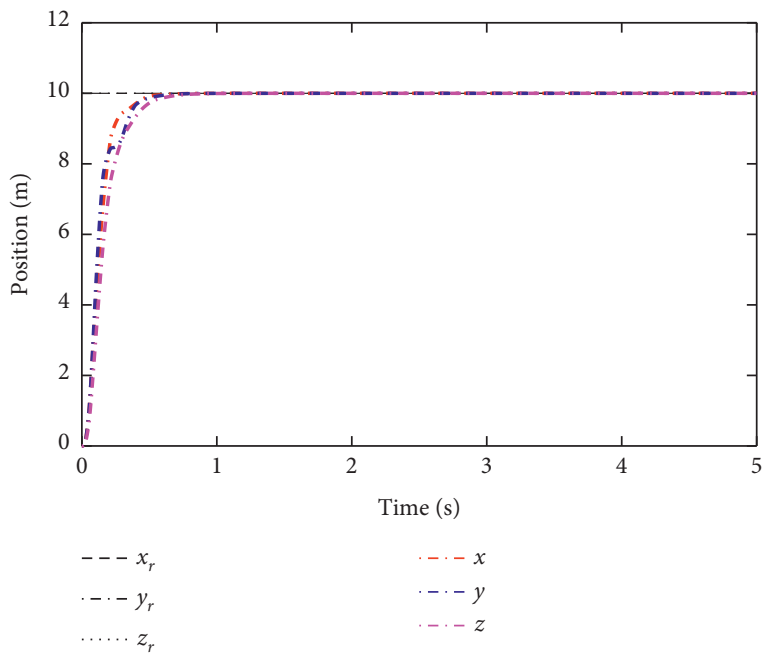

Figure 7: Position response under step input.

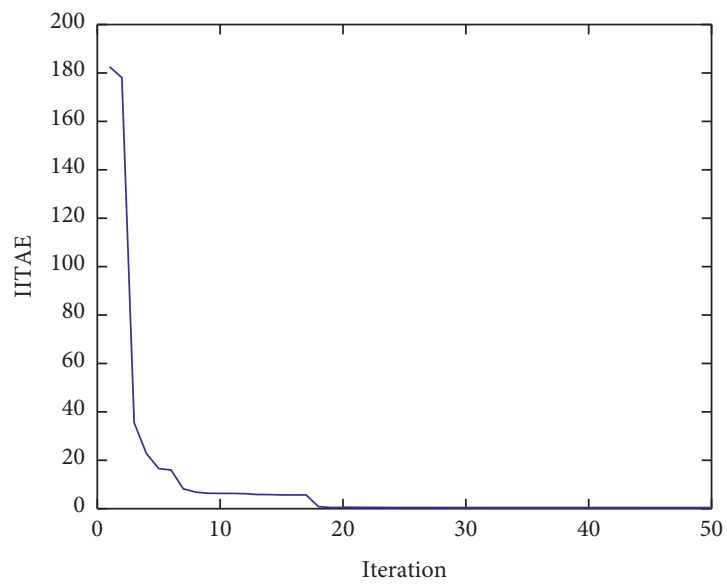

FIgURE 8: Iteration curve of the LADRC of the position loop.

TABLE 1: Tuned control parameters of the LADRC-CISMC.

\begin{tabular}{lccc}
\hline Parameters & Value & Parameters & Value \\
\hline$\omega_{o-x}$ & 58.06 & $\omega_{c-x}$ & 677.72 \\
$\omega_{o-y}$ & 41.02 & $\omega_{c-y}$ & 520.10 \\
$\omega_{o-z}$ & 16.45 & $\omega_{c-z}$ & 553.41 \\
$k_{0}^{\phi}$ & 1.47 & $k_{1}^{\phi}$ & 4.69 \\
$k_{\phi}$ & 64 & $\mu_{\phi}$ & 0.93 \\
$k_{0}^{\theta}$ & 1.25 & $k_{1}^{\theta}$ & 2.78 \\
$k_{\theta}$ & 77 & $\mu_{\theta}$ & 0.82 \\
$k_{0}^{\psi}$ & 1.77 & $k_{1}^{\psi}$ & 7.19 \\
$k_{\psi}$ & 53 & $\mu_{\psi}$ & 0.41 \\
\hline
\end{tabular}

our proposed controller. As depicted in Figure 9, the experimental platform includes an X450 quadrotor, a remote controller, a ground operational station, a global positioning system (GPS), and a flight controller called Pixhawk. The quadrotor is equipped with a flight controller that includes an embedded processor, sensors and propulsion components, and a wireless data link connected to a device capable of transmitting data wirelessly. Ground station programs process flight data for information extraction and retrace data regarding the quadrotor's status. The quadrotor's actual position is collected by GPS. Meanwhile, the quadrotor adopted HOBBYWING eagle 20A electronic speed controllers (ESC), SUNNYSKY A2212-980 kV brushless motors, $2200 \mathrm{mAh}$ lithium battery, and 9450 carbon fibre propellers. The experimental data are collected at a frequency of $50 \mathrm{~Hz}$, which is transformed into the ground operational station. The proposed hybrid controller has been embedded into the Pixhawk hardware from the simulation platform by a plug-in named PX4 autopilots support [29].

We use a Lissajous curve in the form of [ $\sin (0.5 t), \sin (0.25 t), 9.5] \mathrm{m}[30]$ as the reference tracking trajectory. The wind gusts are measured by an AR816 digital anemometer, and the maximum gust measured in the experiments is $3.5 \mathrm{~m} / \mathrm{s}$. Meanwhile, the performance of the proposed LADRC-CISMC compared to the PID is discussed. The optimized control parameters of the PID are listed in Table 2. Figures 10-16 depict the results of 20 


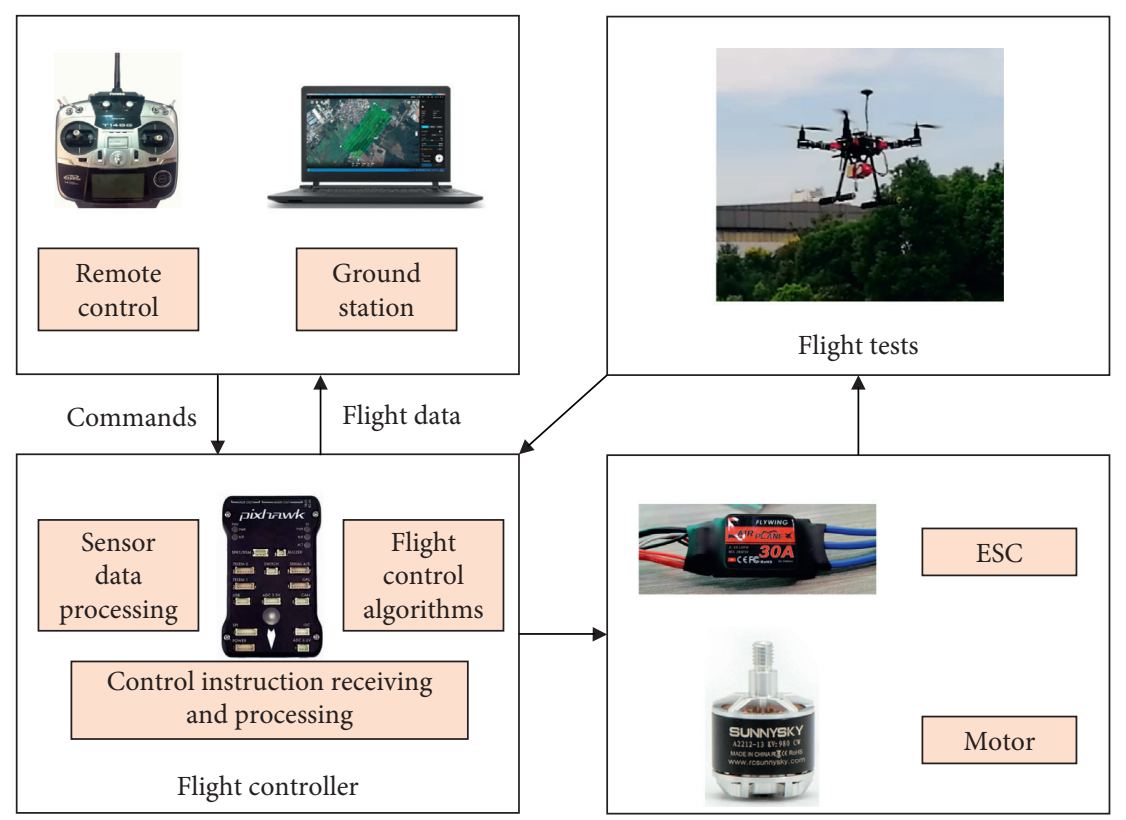

FIGURE 9: Frame of the experimental platform.

seconds of experimental data collection (LADRC-CISMC is called controller 1; PID is called controller 2). It is shown in Figure 10 that the quadrotor can follow a predetermined trajectory. However, the position controlled by PID fluctuates obviously compared to the hybrid controller in the presence of the lumped disturbances. It indicates that the PID control method has no disturbance rejection ability. Due to the delay in GPS signals, the actual position is slightly different from the predetermined trajectory. Nevertheless, our controller performs better than PID. Figures 11-16 show the behavior of the quadrotor's pose, and it is evident a reduction in position and Euler angles amplitude in our proposed controller compared to PID.

Furthermore, two evaluation functions of maximum error $e_{\max }$ and mean square error $e_{\text {mse }}[31,32]$ are proposed to evaluate the performance of the LADRC-CISMC (controller 1) and the LADRC (controller 2), which are described as follows:

$$
\left\{\begin{array}{l}
e_{\max }=\max \left(\left|l_{i}\right|\right), \\
e_{\mathrm{mse}}=\sqrt{\frac{1}{N} \sum_{i=1}^{N}\left(l_{i}\right)^{2},}
\end{array}\right.
$$

where $l_{i}$ is the gap between the referenced and experimental trajectories at $i$ sampling time and $N$ is the sampling length. The calculated results are listed in Table 3. The maximum error value of controller 1 in the position loop is $67.68 \%, 87.56 \%$, and $22.17 \%$ less than that of controller 2, respectively. The mean square error of controller 1 in the position loop is $55.67 \%, 87.56 \%$, and
TABLE 2: Tuned control parameters of the LADRC.

\begin{tabular}{lccc}
\hline Parameters & Value & Parameters & Value \\
\hline$\omega_{o-x}$ & 500.00 & $\omega_{c-x}$ & 54.55 \\
$\omega_{o-y}$ & 499.10 & $\omega_{c-y}$ & 50.70 \\
$\omega_{o-z}$ & 386.45 & $\omega_{c-z}$ & 23.23 \\
$\omega_{o-\phi}$ & 222.78 & $\omega_{c-\phi}$ & 91.67 \\
$\omega_{o-\theta}$ & 310.02 & $\omega_{c-\theta}$ & 95.00 \\
$\omega_{o-\psi}$ & 457.24 & $\omega_{c-\psi}$ & 78.11 \\
\hline
\end{tabular}

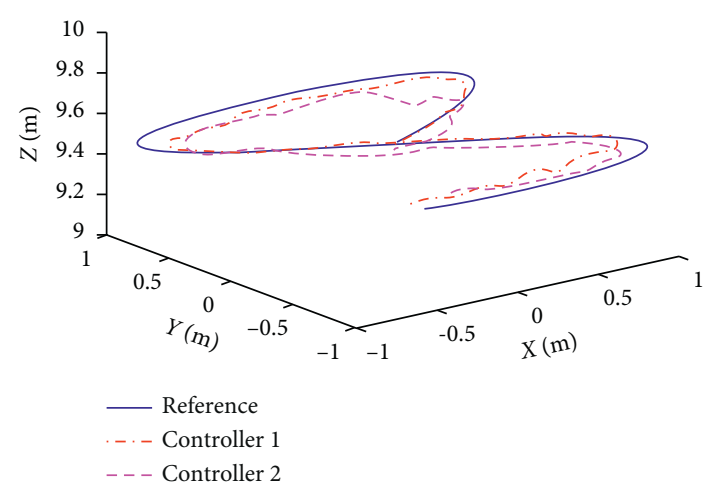

FIgURE 10: 3D tracking trajectory of the quadrotor.

$16.81 \%$ less than that of controller 2 , respectively. It indicates that the proposed controller has a higher tracking precision than the LADRC.

It can be concluded from the above comparison experimental results that the LADRC-CISMC can achieve greater performance than PID in trajectory tracking. 


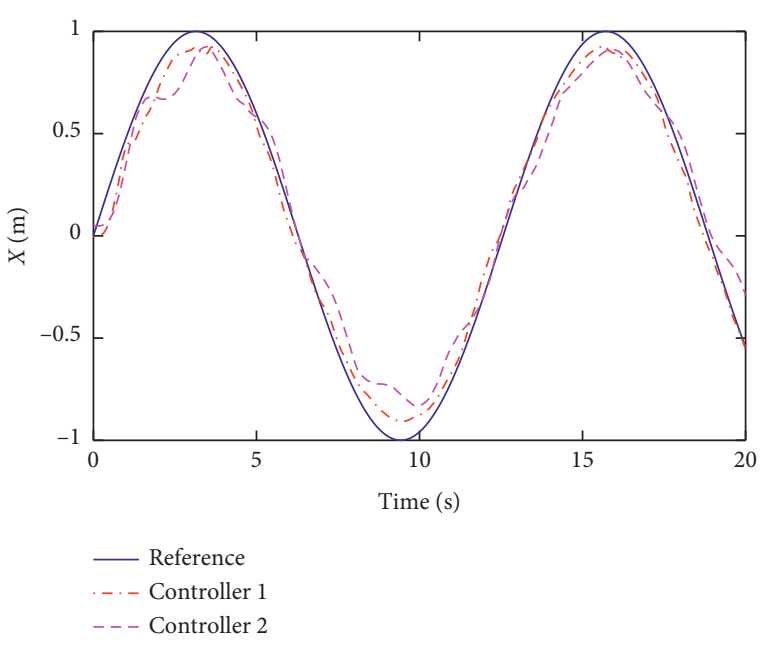

Figure 11: $x$ position quadrotor response.

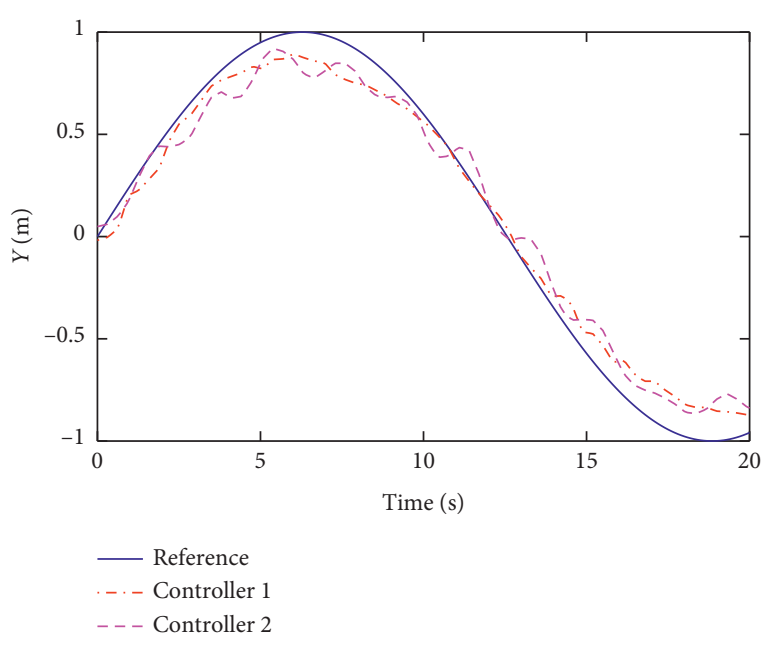

FIGURE 12: $y$ position quadrotor response.

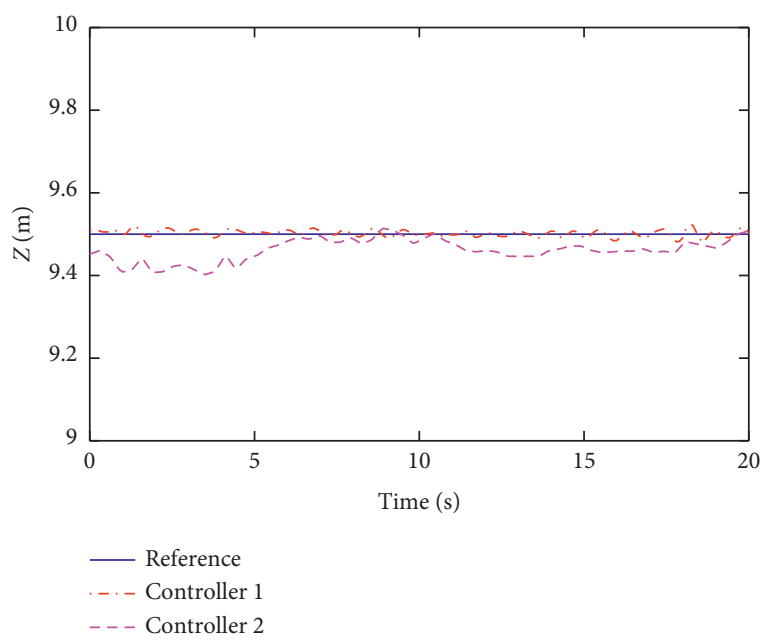

FIGURE 13: $z$ position quadrotor response.

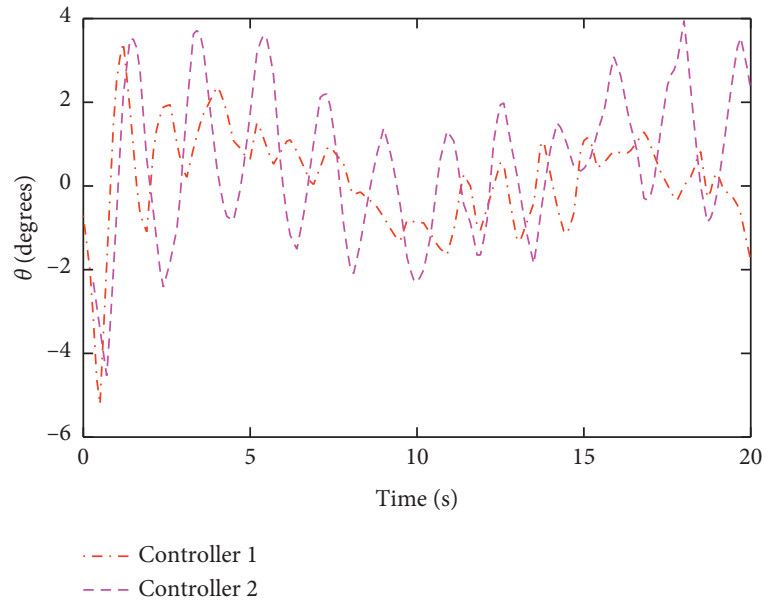

Figure 14: $\theta$ attitude quadrotor response.

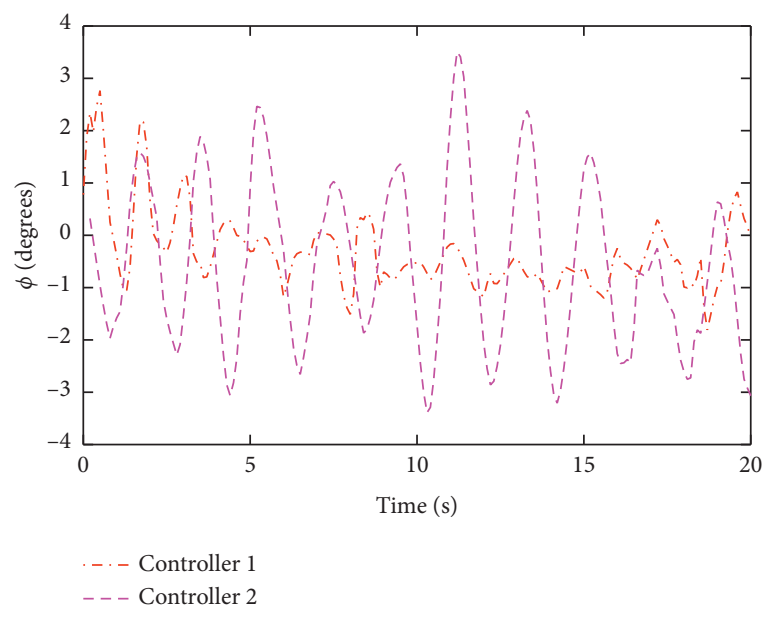

Figure 15: $\phi$ attitude quadrotor response.

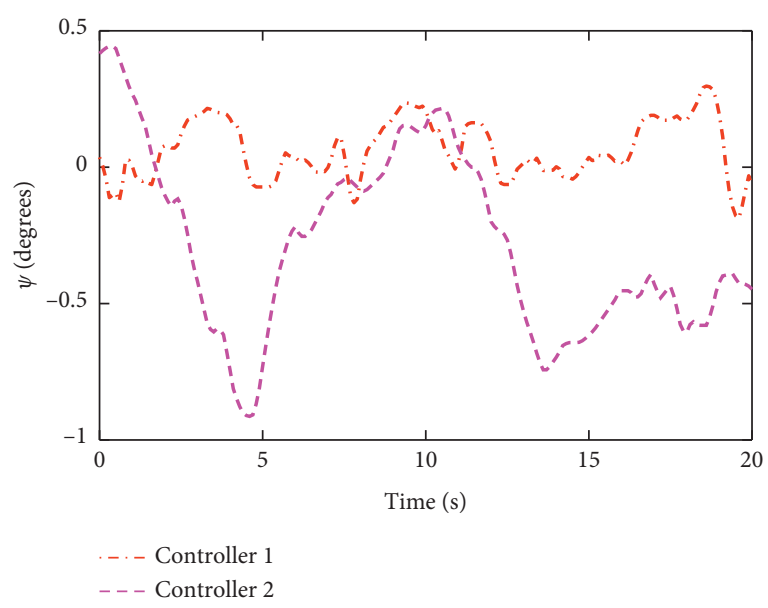

FIGURE 16: $\psi$ attitude quadrotor response. 
TABLE 3: Comparison of tracking errors of the two controllers.

\begin{tabular}{lccc}
\hline \multirow{2}{*}{ State } & \multirow{2}{*}{ Index } & \multicolumn{2}{c}{ Controller } \\
& & Controller 1 & Controller 2 \\
\hline \multirow{2}{*}{$x$} & $e_{\max }$ & 0.1734 & 0.2562 \\
& $e_{\operatorname{mse}}$ & 0.0736 & 0.1322 \\
\hline \multirow{2}{*}{$y$} & $e_{\max }$ & 0.1970 & 0.2250 \\
& $e_{\operatorname{mse}}$ & 0.1105 & 0.1262 \\
\hline \multirow{2}{*}{$z$} & $e_{\max }$ & 0.0217 & 0.0979 \\
& $e_{\operatorname{mse}}$ & 0.0079 & 0.0470 \\
\hline
\end{tabular}

Moreover, the proposed hybrid controller exhibits a higher ability to reject lumped disturbances.

\section{Conclusions}

The issue of trajectory tracking control for quadrotors subjected to lumped disturbances has been investigated in this article. A novel hybrid controller is presented for the dual-loop system. On the basis of this design, a robust controller named LADRC is proposed for the position loop, and a conditional integrator sliding mode controller is designed for the attitude loop. The proposed controller provides high precision and a fast convergence rate for the proposed control strategy since the quadrotor is subjected to lumped disturbances. Moreover, Lyapunov theory is used to analyze the stability of a closed-loop system. Simulation and experimental results confirm the effectiveness and robustness of the proposed hybrid controller LADRC-CISMC. Our future works will focus on the fault-tolerant control for the quadrotor.

\section{Symbols}

$\begin{array}{ll}F_{j}(j=1 \sim 4): & \text { Propeller thrust } \\ k_{t}: & \text { Thrust coefficient } \\ M_{j}(j=1 \sim 4): & \text { Propeller moment } \\ k_{m}: & \text { Moment coefficient } \\ \omega_{j}(j=1 \sim 4): & \text { Propeller speed } \\ \rho_{a}: & \text { Density of air } \\ \tau=\left[\tau_{1}, \tau_{2}, \tau_{3}, \tau_{4}\right]^{T}: & \text { Control torque } \\ R: & \text { Radius of the propeller } \\ \mathbf{q}=[\phi, \theta, \psi]^{T}: & \text { Euler angle } \\ m: & \text { Mass of the aircraft } \\ \mathbf{p}=[x, y, z]^{T}: & \text { Position } \\ g: & \text { Gravitational acceleration } \\ \mathbf{I}=\operatorname{diag}\left(I_{x}, I_{y}, I_{z}\right): & \text { Rotational inertia } \\ A: & \text { Disk area of propeller } \\ k_{x}, k_{y}, k_{z}: & \text { Air drag coefficient } \\ L: & \text { Distance between the center of the } \\ \omega_{o}: & \text { propeller hub } \\ k_{0} \text { and } k_{1}: & \text { Observer bandwidth } \\ \omega_{c}: & \text { Positive numbers } \\ \sigma: & \text { Control bandwidth } \\ k: & \text { Conditional integrator } \\ \mu: & \text { Control gain } \\ \rho: & \text { Parameter } \\ \mathbf{L}_{s}: & \text { Positive number } \\ & \text { Observer gain } \\ & \end{array}$

$\begin{array}{ll}u_{0}: & \text { Error feedback } \\ t_{s}: & \text { Settling time } \\ o_{s}: & \text { Overshoot } \\ \alpha_{1}, \alpha_{2}, \alpha_{3}, \alpha_{4}: & \text { Weight factors. }\end{array}$

\section{Data Availability}

The data used to support the findings of this study are available from the corresponding author upon request.

\section{Conflicts of Interest}

The authors declare that they have no conflicts of interest.

\section{References}

[1] W. Hönig, J. A. Preiss, T. K. S. Kumar, G. S. Sukhatme, and N. Ayanian, "Trajectory planning for quadrotor swarms," IEEE Transactions on Robotics, vol. 34, no. 4, pp. 856-869, 2018.

[2] M. Labbadi and M. Cherkaoui, "Robust adaptive backstepping fast terminal sliding mode controller for uncertain quadrotor UAV," Aerospace Science and Technology, vol. 93, Article ID 105306, 2019.

[3] M. Bucolo, A. Buscarino, C. Famoso, L. Fortuna, and M. Frasca, "Control of imperfect dynamical systems," Nonlinear Dynamics, vol. 98, no. 4, pp. 2989-2999, 2019.

[4] N. Imanberdiyev and E. Kayacan, "A fast learning control strategy for unmanned aerial manipulators," Journal of Intelligent \& Robotic Systems, vol. 94, no. 3, pp. 805-824, 2019.

[5] S. Bouabdallah, A. Noth, and R. Siegwart, "PID vs LQ control techniques applied to an indoor micro quadrotor," IEEE in Proceedings of the 2004 IEEE/RSJ International Conference on Intelligent Robots and Systems (IROS), vol. 3, pp. 2451-2456, Sendai, Japan, September 2004.

[6] D. Ma, Y. Xia, T. Li, and K. Chang, "Active disturbance rejection and predictive control strategy for a quadrotor helicopter," IET Control Theory \& Applications, vol. 10, no. 17, pp. 2213-2222, 2016.

[7] L. D. Minh and C. Ha, "Modeling and control of quadrotor MAV using vision-based measurement," in Proceedings of the International Forum on Strategic Technology 2010, pp. 70-75, IEEE, Ulsan, South Korea, October 2010.

[8] U. Ansari and A. H. Bajodah, "Robust generalized dynamic inversion quadrotor control," IFAC-PapersOnLine, vol. 50, no. 1, pp. 8181-8188, 2017.

[9] B. Tian, L. Liu, H. Lu et al., "Multivariable finite time attitude control for quadrotor UAV: theory and experimentation," IEEE Transactions on Industrial Electronics, vol. 65, no. 3, pp. 2567-2577, 2017.

[10] W. Dong, G.-Y. Gu, X. Zhu, and H. Ding, "High-performance trajectory tracking control of a quadrotor with disturbance observer," Sensors and Actuators A: Physical, vol. 211, pp. 67-77, 2014.

[11] A. A. Najm and I. K. Ibraheem, "Altitude and attitude stabilization of UAV quadrotor system using improved active disturbance rejection control," Arabian Journal for Science and Engineering, vol. 45, no. 3, pp. 1985-1999, 2020.

[12] L. Ding, J. Zhou, and W. Shan, "A hybrid high-performance trajectory tracking controller for unmanned hexrotor with disturbance rejection," Transactions of the Canadian Society for Mechanical Engineering, vol. 42, no. 3, pp. 239-251, 2018. 
[13] M. A. Mohd Basri, A. R. Husain, and K. A. Danapalasingam, "A hybrid optimal backstepping and adaptive fuzzy control for autonomous quadrotor helicopter with time-varying disturbance," Proceedings of the Institution of Mechanical Engineers, Part G: Journal of Aerospace Engineering, vol. 229, no. 12, pp. 2178-2195, 2015.

[14] U. Ansari, A. H. Bajodah, and M. T. Hamayun, "Quadrotor control via robust generalized dynamic inversion and adaptive non-singular terminal sliding mode," Asian Journal of Control, vol. 4, no. 21, pp. 1-13, 2019.

[15] S. Bouabdallah, P. Murrieri, and R. Siegwart, "Design and control of an indoor micro quadrotor," in Proceedings of the 2004 IEEE International Conference on Robotics and Automation, vol. 5, pp. 4393-4398, New Orleans, LA, USA, June 2004.

[16] W. Dong, G. Y. Gu, X. Zhu et al., "Modeling and control of a quadrotor UAV with aerodynamic concepts," World Academy of Science, Engineering and Technology (WASET), vol. 7, pp. 437-448, 2013.

[17] J. Zhao, H. Zhang, and X. Li, “Active disturbance rejection switching control of quadrotor based on robust differentiator," Systems Science \& Control Engineering, vol. 8, no. 1, pp. 605-617, 2020.

[18] H. L. N. Ngoc Thanh and S. K. Hong, "An extended multisurface sliding control for matched/mismatched uncertain nonlinear systems through a lumped disturbance estimator," IEEE Access, vol. 8, pp. 91468-91475, 2020.

[19] J. Zhang, J. Feng, Y. Zhou, F. Fang, and H. Yue, "Linear active disturbance rejection control of waste heat recovery systems with organic rankine cycles," Energies, vol. 5, no. 12, pp. 5111-5125, 2012.

[20] Z. Gao, "Active disturbance rejection control: a paradigm shift in feedback control system design," IEEE, in Proceedings of the 2006 American Control Conference, pp. 2399-2405, Minneapolis, MN, USA, June 2006.

[21] H. Niu, Q. Gao, S. Tang et al., "Linear active disturbance rejection control for lever-type electric erection system based on approximate model," Journal of Control Science and Engineering, vol. 2019, Article ID 3742694, 7 pages, 2019.

[22] Y. Liu, M. Lu, X. Wei et al., "Introducing conditional integrator to sliding mode control of $\mathrm{dc} / \mathrm{dc}$ buck converter," in Proceedings of the 2009 IEEE International Conference on Control and Automation, pp. 891-896, IEEE, Christchurch, New Zealand, December 2009.

[23] Z. Shi, C. Deng, S. Zhang, Y. Xie, H. Cui, and Y. Hao, "Hyperbolic tangent function-based finite-time sliding mode control for spacecraft rendezvous maneuver without chattering," IEEE Access, vol. 8, pp. 60838-60849, 2020.

[24] J. Qian, C. Ji, N. Pan, and J. Wu, "Improved sliding mode control for permanent magnet synchronous motor speed regulation system," Applied Sciences, vol. 8, no. 12, pp. 2491-2502, 2018.

[25] Y. Liu, X. Li, T. Wang, Y. Zhang, and P. Mei, "Quantitative stability of quadrotor unmanned aerial vehicles," Nonlinear Dynamics, vol. 87, no. 3, pp. 1819-1833, 2017.

[26] F. Cairone and M. Bucolo, "Data-driven identification of twophase microfluidic flows," in Proceedings of the 2016 24th mediterranean conference on control and automation (MED), pp. 797-802, IEEE, Athens, Greece, June 2016.

[27] L. Ding and Y. Li, "Optimal attitude tracking control for an unmanned aerial quadrotor under lumped disturbances," International Journal of Micro Air Vehicles, vol. 12, pp. 1-12, 2020 .
[28] R. Ma, L. Ding, and H. Wu, "Dynamic decoupling control optimization for a small-scale unmanned helicopter," Journal of Robotics, vol. 2018, Article ID 9897684, 12 pages, 2018.

[29] Q. Quan, X. Dai, and S. Wang, Multicopter Design and Control Practice: A Series Experiments Based on MATLAB and Pixhawk, Springer Nature, Singapore, 2020.

[30] J. Ren, Y. Ye, G. Xu et al., "Uncertainty-and-disturbanceestimator-based current control scheme for PMSM drives with a simple parameter tuning algorithm," IEEE Transactions on Power Electronics, vol. 32, no. 7, pp. 5712-5722, 2016.

[31] Y. Wang, B. Li, F. Yan, and B. Chen, "Practical adaptive fractional-order nonsingular terminal sliding mode control for a cable-driven manipulator," International Journal of Robust and Nonlinear Control, vol. 29, no. 5, pp. 1396-1417, 2019.

[32] A. Aboudonia, A. El-Badawy, and R. Rashad, "Disturbance observer-based feedback linearization control of an unmanned quadrotor helicopter," Proceedings of the Institution of Mechanical Engineers, Part I: Journal of Systems and Control Engineering, vol. 230, no. 9, pp. 877-891, 2016. 